\title{
Fauna silvestre y productos derivados decomisados durante el período 2000-2007, Lima - Perú
}

Wildlife and wildlife products confiscated during the period 2000-2007, Lima - Peru

\author{
Nathaly Quevans ${ }^{1}$, Néstor Falcón ${ }^{1}$, Roberto Elías ${ }^{1}$ \\ RESUMEN
}

Objetivos: Describir las características del decomiso de animales silvestres y productos derivados realizado por la Administración Técnica Forestal y de Fauna Silvestre (ATFFS) durante el período 2000 - 2007 en la ciudad de Lima - Perú. Material y métodos: Para ello, se revisó la base de datos de decomisos de la ATFFS para el período de estudio. Resultados: se encontró 17932 animales vivos y 81564 productos derivados decomisados en el período 2000-2007. Entre los animales vivos, el 47,3\% (8479) fueron aves, 34,1\% (6111) anfibios, 13,1\% (2341) reptiles y $5,6 \%$ (1001) mamíferos. La mayor cantidad de especies decomisadas corresponde a las aves de la familia Psittacidae. De los productos derivados de animales silvestres, el 98,3\% (80216) correspondió a animales taxidermizados, $0,8 \%$ (627) pieles, $0,7 \%$ (600) instrumentos y $0,1 \%$ (121) carnes. También se encontró ejemplares no identificados lo que no permite obtener información necesaria para la evaluación de la situación actual de las especies sujetas a tráfico ilegal. Conclusiones: Debido a la magnitud del tráfico de animales silvestres y productos derivados se hace necesario buscar nuevas alternativas para reducir este tipo de actividad ilícita que afecta a la biodiversidad del país.

PALABRAS CLAVE: Fauna silvestre, decomisos, ATFFS, tráfico ilegal de animales

\section{SUMMARY}

Objectives: To describe the characteristics of the confiscation wildlife and products made by the Technical Administration of Forestry and Wildlife (ATFFS) during the period from 2000 to 2007 in the city of Lima - Peru. Methods: To do this, we reviewed the database ATFFS for the study period Results: 17932 live animals and 81564 products confiscated in the period 2000-2007. Among living animals, 47.3\% (8479) were birds, 34.1\% (6111) amphibian, 13.1\% (2341) Reptiles and 5,6\% (1001) mammals. Most species of birds confiscated corresponds to family Psittacidae. Of wildlife products, $98.3 \%$ (80216) corresponded to animal taxidermy, $0.8 \%$ (627) furs, $0.7 \%$ (600) instruments and $0.1 \%$ (121) bush meat. Also found an unidentified specimen which does not allow obtaining information necessary for the evaluation of the current status of the species subject to trade. Conclusions: Because of the extent of trade of wildlife and their products is necessary to find new ways to reduce this kind of illegal activity affecting the country's biodiversity.

KEYWORDS: Wildlife, confiscations, ATFFS, ilegal animal trade.

Facultad de Medicina Veterinaria y Zootecnia, Universidad Peruana Cayetano Heredia. Lima, Perú. 


\section{INTRODUCCIÓN}

El tráfico ilegal de animales es una de las mayores causas de pérdida de fauna en el mundo. Los animales silvestres son cazados para ser comercializados como mascotas, para consumo, por supuestas propiedades para la medicina y brujería/afrodisíaco o comercio de sus derivados (1-3).

Según datos de la Secretaría de la Convención sobre el Comercio Internacional de Especies Amenazadas de Fauna y Flora Silvestres (CITES), tres zonas destacan como fuentes de extracción de animales silvestres: África, América del Sur y el sud-este de Asia (4). Los principales mercados que demandan animales silvestres se sitúan en los países ricos, siendo los compradores más importantes países como Japón, Estados Unidos y los de la Unión Europea (5).

El Perú es uno de los mayores centros mundiales de recursos genéticos y por ello suele ser requerido para fines comerciales. El 80\% de animales silvestres comercializados en Lima proviene de la región San Martín y de las ciudades de Iquitos, Pucallpa y Puerto Maldonado. El comercio de estos animales está penado según la Ley 27308 "Ley Forestal y de Fauna Silvestre", en donde se establece que las personas que comercialicen y adquieran ilegalmente especies silvestres, de flora y fauna, podrán ser sancionadas con cinco años de cárcel y con multas superiores a los dos millones de nuevos soles (600 Unidades Impositivas Tributarias) (6).

La tenencia de animales silvestres como mascotas es una costumbre con arraigos culturales que en la actualidad ha contribuido como un factor que favorece el tráfico de estos animales. Nuestros antepasados los veían como símbolos y por ello los mantenían en cautiverio (7). Parece que una actitud humanista y estética, asociada a empatía malinterpretada y un sentimiento afectivo hacia los animales, puede ser un origen de la motivación a tener un animal cautivo (8).

Una encuesta realizada en Costa Rica encuentra que uno de cada cuatro hogares poseía un animal silvestre como mascota, siendo el taxón más afectado los psitácidos. Las causas del mismo son por estética y por el placer que produce su tenencia, siendo la mayoría de los animales entregados como regalo o como una forma de estimular el amor por la naturaleza a los niños (9). Esta práctica alcanza inclusive a estudiantes de Medicina Veterinaria quienes poseen animales silvestres como mascota, independiente del conocimiento de las regulaciones legales que se tienen al respecto (10).

En nuestro país está permitido la tenencia de animales silvestres como mascotas, sólo si estos provienen de instituciones legales, siendo esta una modalidad sin fines comerciales de manejo y aprovechamiento de la fauna silvestre que es aceptada por la Ley Forestal y de Fauna Silvestre, en donde son divididas en dos: las que tienen fines comerciales, donde podemos encontrar a los zoocriaderos, áreas de manejo de fauna silvestre y cotos de caza, y las que son sin fines comerciales, como zoológicos, centros de rescate, centros de custodia temporal, animales silvestres como mascota (6)

Debido a la importancia que representa el tráfico ilegal de animales silvestres se hace necesario visualizar, aunque parcialmente, la magnitud del mismo en Lima a través de la información obtenida de los decomisos de especies. Por ello el objetivo del estudio fue caracterizar el decomiso de animales silvestres y productos derivados realizado por ATFFS durante el período 2000 - 2007 en la ciudad de Lima Perú.

\section{MATERIAL Y MÉTODOS}

La información de los decomisos fue solicitada a las oficinas de la Administración Técnica Forestal y de Fauna Silvestre (ATFFS) de Lima - ubicada en Jr. Huamachuco 1833, Jesús María. La base de datos y el análisis de la misma fueron realizadas en la Facultad de Medicina Veterinaria y Zootecnia de la Universidad Peruana Cayetano Heredia (FAVEZ-UPCH).

La información de la población en estudio estuvo limitada por la base de datos de decomisos de la ATFFS para el período 2000-2007. A partir de la misma se determinaron las siguientes variables de estudio:

- Especies de fauna silvestre decomisados.

- Taxones a las que pertenecen las especies decomisadas.

- Número de ejemplares decomisados.

- Tipo de decomiso (animal vivo o producto derivado).

Se elaboró una nueva base en el programa Microsoft Excel y el análisis de datos se realizó utilizando el programa SPSS 17.0. Los datos fueron resumidos mediante estadística descriptiva a través de tablas de frecuencias. Los resultados se clasificaron de acuerdo al tipo de decomiso del animal (vivo y 
Tabla 1. Distribución de animales silvestres (según clase) y productos derivados decomisados durante el período 2002-2007 en Lima - Perú.

\begin{tabular}{cccc}
\hline $\begin{array}{c}\text { Tipo de } \\
\text { decomiso }\end{array}$ & Clasificación & Número & Porcentaje \\
\hline \multirow{2}{*}{ Animales vivos } & Reptiles & 2341 & 13,1 \\
& Aves & 8479 & 47,3 \\
& Mamíferos & 1001 & 5,6 \\
& TOTAL & $\mathbf{1 7 9 3 2}$ & $\mathbf{1 0 0}$ \\
\hline \multirow{2}{*}{$\begin{array}{c}\text { Productos } \\
\text { derivados }\end{array}$} & Paxidermizados & 80216 & 98,3 \\
& Instrumentos & 600 & 0,8 \\
& Carnes & 121 & 0,7 \\
& TOTAL & $\mathbf{8 1 5 6 4}$ & $\mathbf{1 0 0}$ \\
\hline
\end{tabular}

Tabla 2. Animales según clase y familia decomisados con mayor frecuencia durante el período 2000-2007 en Lima Perú.

\begin{tabular}{|c|c|c|c|}
\hline Clase & Familia & $\mathbf{N}$ & $\%$ \\
\hline \multirow{5}{*}{ ANFIBIOS } & Dendrobatidae & 400 & 6,6 \\
\hline & Ceratophryidae & 334 & 5,5 \\
\hline & No identificado & 5050 & 82,6 \\
\hline & Otros & 327 & 5,3 \\
\hline & TOTAL & 6111 & 100,0 \\
\hline \multirow{6}{*}{ REPTILES } & Iguanidae & 778 & 33,2 \\
\hline & Testunidae & 557 & 23,8 \\
\hline & Podocnemididae & 274 & 11,7 \\
\hline & No identificado & 564 & 24,1 \\
\hline & Otros & 168 & 7,2 \\
\hline & TOTAL & 2341 & 100,0 \\
\hline \multirow{8}{*}{ AVES } & Psittacidae & 3453 & 40,7 \\
\hline & Fringillidae & 1340 & 15,8 \\
\hline & Emberizidae & 826 & 9,7 \\
\hline & Tharupidae & 233 & 2,8 \\
\hline & Icteridae & 219 & 2,6 \\
\hline & No identificado & 2060 & 24,3 \\
\hline & Otros & 348 & 4,1 \\
\hline & TOTAL & 8479 & 100,0 \\
\hline \multirow{9}{*}{ MAMIFEROS } & Cebidae & 387 & 38,7 \\
\hline & Sciuridae & 120 & 12,0 \\
\hline & Atelidae & 117 & 11,7 \\
\hline & Procyonidae & 74 & 7,4 \\
\hline & Felidae & 51 & 5,1 \\
\hline & Callitricidae & 49 & 4,9 \\
\hline & No identificado & 68 & 6,8 \\
\hline & Otros & 135 & 13,5 \\
\hline & TOTAL & 1001 & 100,0 \\
\hline
\end{tabular}

Tabla 3. Vertebrados taxidermizados decomisados durante el período 2000-2007 en Lima-Perú.

\begin{tabular}{|c|c|c|c|}
\hline Tipo & Nombre común & $\mathrm{N}$ & $\%$ \\
\hline \multirow{11}{*}{ Aves } & Aguilucho & 3 & 0,44 \\
\hline & Búho & 1 & 0,15 \\
\hline & Tangara & 21 & 3,11 \\
\hline & Paca & 1 & 0,15 \\
\hline & Picaflor & 2 & 0,3 \\
\hline & Loro & 3 & 0,44 \\
\hline & Pato & 22 & 3,25 \\
\hline & Tucán & 4 & 0,59 \\
\hline & Tucaneta & 3 & 0,44 \\
\hline & No identificado & 11 & 1,63 \\
\hline & SUB TOTAL & 71 & 10,5 \\
\hline \multirow{8}{*}{ Mamíferos } & Ardilla & 4 & 0,59 \\
\hline & Armadillo & 34 & 5,03 \\
\hline & Murciélago & 285 & 42,16 \\
\hline & Mono & 7 & 1,04 \\
\hline & Venado & 3 & 0,44 \\
\hline & Zorro & 16 & 2,37 \\
\hline & No identificado & 3 & 0,44 \\
\hline & SUB TOTAL & 352 & 52,07 \\
\hline \multirow{7}{*}{ Reptiles } & Caimán & 40 & 5,92 \\
\hline & Lagarto & 48 & 7,1 \\
\hline & Tortugas & 4 & 0,59 \\
\hline & Lagartija & 28 & 4,14 \\
\hline & Iguanas & 81 & 11,98 \\
\hline & Boa & 4 & 0,59 \\
\hline & SUB TOTAL & 205 & 30,33 \\
\hline Anfibios & Sin definir & 1 & 0,15 \\
\hline No identificado & Sin definir & 47 & 6,95 \\
\hline \multicolumn{2}{|c|}{ Total de decomisos } & 676 & $100,00 \%$ \\
\hline
\end{tabular}

productos derivados) y para cada grupo se cuantificó el número de ejemplares decomisados.

\section{RESULTADOS}

El estudio obtuvo un total de 17932 animales vivos y 81564 productos derivados decomisados durante el período 2000-2007. El 47,8\% (8 479) pertenecía a la clase Aves dentro de la clasificación de animales vivos decomisados en mayor número, seguido de la clase Amphibia con 34,1\% (6111) Entre los productos derivados predominó los animales taxidermizados representando el 98,1\% (80 216) del total. El detalle se presenta en la tabla 1. La distribución, según clase y familia, de la cantidad de animales vivos decomisados durante el período de estudio se detalla en la tabla 2. 
Tabla 4. Invertebrados taxidermizados decomisados durante el período 2007-2007 en Lima-Perú.

\begin{tabular}{cccc}
\hline Tipo & Nombre común & $\mathrm{N}$ & $\%$ \\
\hline \multirow{2}{*}{ Arácnidos } & Escorpiones & 25 & 0,02 \\
& Tarántulas & 535 & 0,34 \\
& Arañas & 15 & 0,01 \\
& SUB TOTAL & $\mathbf{5 7 5}$ & $\mathbf{0 , 3 6}$ \\
\hline \multirow{2}{*}{ Lepidópteros } & Mariposa & 69846 & 43,91 \\
& No identificados & 191 & 0,12 \\
Coleópteros & SUB TOTAL & $\mathbf{7 0 ~ 0 3 7}$ & $\mathbf{4 4 , 0 3}$ \\
\hline Ortópteros & Escarabajos & 6770 & 4,26 \\
Formícidos & Saltamontes & 208 & 0,13 \\
Anisópteros & Hormigas & 1937 & 1,22 \\
& Libélulas & 13 & 0,01 \\
& SUB TOTAL & $\mathbf{8 9 2 8}$ & $\mathbf{5 , 6 1}$ \\
\hline & Total & $\mathbf{7 9 5 4 0}$ & $\mathbf{1 0 0 , 0 \%}$ \\
\hline
\end{tabular}

El total de animales vertebrados taxidermizados ascendió a 676 ejemplares. De ellos 71 eran aves 352 mamíferos, 205 reptiles, un anfibio y 47 decomisos de los cuales no se obtuvo ninguna información, el detalle se observa en la tabla 3.

El total de invertebrados taxidermizados fue de 70540 ejemplares. De ellos 575 eran arácnidos y 70 037 lepidópteros, También se encontraron coleópteros (6 770 escarabajos), ortópteros (208 saltamontes), formícidos (1 937 hormigas) y anisópteros (13 libélulas), el detalle se observa en la tabla 4.

\section{DISCUSIÓN}

El estudio investigó el número de animales silvestres y productos derivados decomisados por la ATFFS durante un período de siete años en la ciudad de Lima y de acuerdo a los resultados encontrados se puede inferir que este problema es importante en el Perú, tan igual como lo deben sufrir otros países con alta diversidad biológica. La Resolución Jefatural $\mathrm{N}^{\circ}$ 065-2000 de INRENA menciona acerca del comercio de artesanías y despojos de fauna silvestre e indica que solo se puede hacer uso de estos subproductos si provienen de la caza de subsistencia o si no se encuentran en alguna lista de riesgo (11).

La magnitud de este problema es tan importante que se convierte en el tercer comercio ilegal después del tráfico de drogas y armas. La demanda de animales silvestres y productos derivados varía en el tiempo, siendo una constante que aves y productos taxidermizados sean los más solicitados $(1,12)$. Estas variaciones se puede deber a la demanda del mercado y la capacidad de poder encontrar la especie, es por eso que en la mayoría de los casos se puede confirmar los resultados en otros estudios, donde el uso de los animales silvestres va depender de características sociales, políticas y culturales de cada país, que se considera una estrategia por parte de los traficantes de fauna que impulsan el consumo de determinadas especies y subproductos de la fauna que tiene una alta demanda en el mercado interno (3).

Se ha asociado también a la tenencia de animales silvestres como mascota como un factor que favorece la demanda de estas especies. La tenencia de animales silvestres ha ido en aumento con el pasar de los años, siendo las causas más comunes su atractivo estético y el placer que causa su tenencia (12). En este rubro, serían las aves las más solicitadas según los resultados del estudio y su tráfico se ve favorecido por el tamaño de los animales y lo fácil que resulta su transporte. Estas son transportadas en cajas, baldes, sacos, botellas, cañas, entre otros tratándolas como objetos y siendo producto de maltrato que se refleja en una alta mortalidad a la llegada al destino (13). El segundo puesto lo encabezan los anfibios, los cuales son cazados por los traficantes y vendidos como mascotas por los colores llamativos que tienen algunas especies que son solicitadas por coleccionistas, o también por requerimientos de empresas farmacéuticas (14).

Hay que considerar que una tenencia irresponsable de los animales silvestres como mascota conlleva a problemas que afectan el bienestar animal y pueden comprometer la salud de estos y las personas. Las personas deciden dejar de tener a estos animales como mascota debido a los problemas de conducta y costo de su manutención, además de los problemas de salud del animal y los que pueden producir a sus dueños (3). Similar situación se presenta con los mamíferos, en donde los más atractivos resultan ser los primates, en particular de la Familia Cebidae, por su fácil adaptación a las costumbres humanas.

El control del tráfico de animales silvestres requiere el apoyo de Gobiernos Regionales, Fuerzas Armadas, Policía Nacional, Ministerio Público, SUNAT y OSINFOR, para tener un mayor control del mismo. Sin embargo, se debería dar mayor importancia a las normas que regulan el tráfico, así como incentivar un mayor número de campañas educativas que refuercen el valor y respeto hacia la fauna silvestre. Se hace necesario desarrollar una conciencia acerca de la 
importancia de conservar la biodiversidad como un bien social nacional y mundial.

Ante esto, se hace evidente la importancia del profesional veterinario en el desarrollo de lineamientos técnicos que sirvan de ayuda en la toma de decisiones para determinar el destino de los animales decomisados o hallados en abandono, así como su participación en centros de rescate y de custodia temporal, tanto actuales como futuros.

\section{CONCLUSIONES}

El estudio concluye que el tráfico ilegal de fauna silvestre y sus derivados es una de las principales amenazas de la biodiversidad en el país, siendo los miembros de la familia Psittacidae (Clase Aves) el taxón que sufre la mayor presión por esta actividad en nuestro país. Asimismo, se requiere una mayor capacitación en el personal que realiza los decomisos porque se encontró que un importante número de ejemplares no fueron identificados durante los mismos, lo que no permite obtener información valiosa para seguir evaluando la situación actual de las especies sujetas a tráfico ilegal.

\section{Correspondencia}

Nathaly Quevans Gálvez

Correo electrónico: thalitabella@hotmail.com

\section{REFERENCIAS BIBLIOGRAFICAS}

1. Redford KH, Robinson JG. Subsistence and Commercial Uses of Wildlife in Latin America. En: Robinson JG, Redford KH (Edit). Neotropical wildlife use and conservation. Chicago: University of Chicago Press; 1991.p. 6-23.

2. Ojasti J, Dallmeier F. Manejo de fauna silvestre neotropical. Series \# 5 (Internet). Washington D.C.: Smithsonian Institution/MAB Biodiversity Program; 2000. (Citado en marzo del 2013) Disponible en: http://www.ibcperu.org/doc/isis/13869.pdf

3. Cruz-Antia D, Gómez J. Aproximación al uso y tráfico de fauna silvestre en Puerto Carreño, Vichada, Colombia. Bogotá. Ambiente y desarrollo. 2010; 14(26): 64-94.

4. Camis I, Casanova C, Brizi L. Comercio internacional de especies exóticas. Barcelona, España: Universidad Autónoma de Barcelona; 2010. (Citado en marzo del 2013) Disponible en:

5. http://ddd.uab.cat/pub/trerecpro/2011/80088/ comercio_internacional_de_especies_exoticas_ mercado_negro.pdf

6. Ríos A, Riva F, Canaquire L. Reporte situacional del tráfico ilegal de fauna silvestre en la Región Nororiental del Perú. Lima: Pro naturaleza; 2008. p.72 .

7. Dirección General Forestal y de Fauna Silvestre. Ministerio de agricultura. Notas de prensa. Comercio y compra ilegal de especies silvestres son penados con cárcel y dos millones de soles. Lima. DGFFS; 2011. (Citado en marzo del 2013) Disponible en: http://www.minag.gob.pe/portal/notas-de-prensa/ notas-de-prensa-2011/5060-comercio-y-comprailegal-de-especies-silvestres-son-penados-concarcel-y-dos-millones-de-soles

8. Abarca H. Fauna silvestre en condiciones de cautividad doméstica en Costa Rica: problemática y discusiones. Revista Biocenosis. 2005; 19(2): 31-37.

9. Drews C. Rescate de fauna en el neotrópico: cerrando el milenio. En: C. Drews (ed.). Rescate de fauna en el neotrópico. Heredia, Costa Rica: Editorial Universidad Nacional;1999.p. 495-520.

10. Drews C. Aspectos del mercado en torno a la tenencia de animales silvestres como mascotas en Costa Rica En: Nassar F,Crane R (editores).Actitudes hacia la Fauna en Latinoamérica. Washington, D.C.: Humane Society Press; 2000. p. 147-160.

11. Oroza P. Tenencia de animales no convencionales como mascotas entre estudiantes de la Facultad de Veterinaria y Zootecnia de la Universidad Cayetano Heredia. Tesis de Médico Veterinario Zootecnista. Lima, Perú. Universidad Peruana Cayetano Heredia, 2008. 19pp.

12. INRENA. Resolución Jefatural 065-2000 de INRENA. Lima: Diario Oficial El Peruano; 9 de marzo del 2000.

13. Drews C. Caracterización general de la tenencia de animales silvestres como mascotas en Costa Rica. En: Nassar F, Crane R (editores).Actitudes hacia la Fauna en Latinoamérica. Washington, D.C.: Humane Society Press; 2000. p. 45-55.

14. Baquero MV. Dinámica de la comercialización ilegal de especies de la familia Psittacidae y contexto cultural en las ciudades de Villavicencio, Girardot, Espinal y Bogotá.Tesis de Pregrado. Bogotá, Colombia. Facultad de Ecología, Pontificia Universidad Javeriana, 2005.

15. Rueda A. Anfibios y reptiles amenazados de extinción en Colombia. Rev Acad Colomb Cienc. 1999; 23(Sl): 475-498.

Recibido: 15/03/2013

Aceptado: 24/06/2013 